\title{
Climate change anxiety and mental health: Environmental activism as buffer
}

\author{
Sarah E. O. Schwartz ${ }^{1}$ (1) $\cdot$ Laelia Benoit $^{2,3,4} \cdot$ Susan Clayton $^{5} \cdot$ McKenna F. Parnes $^{1} \cdot$ Lance Swenson $^{1} \cdot$ Sarah R. Lowe ${ }^{6}$
}

Accepted: 16 January 2022

(c) The Author(s), under exclusive licence to Springer Science+Business Media, LLC, part of Springer Nature 2022

\begin{abstract}
A growing body of research has documented the phenomenon of climate change anxiety (CCA), defined broadly as negative cognitive, emotional, and behavioral responses associated with concerns about climate change. A recently validated scale of CCA indicated two subscales: cognitive emotional impairment and functional impairment (Clayton \& Karazsia, 2020). However, there are few empirical studies on CCA to date and little evidence regarding whether CCA is associated with psychiatric symptoms, including symptoms of Major Depressive Disorder (MDD) and Generalized Anxiety Disorder (GAD), and whether engaging in individual and collective action to address climate change could buffer such relationships. This mixed methods study draws on data collected from a sample of emerging adult students (ages 18-35) in the United States $(N=284)$ to address these gaps. Results indicated that both CCA subscales were significantly associated with GAD symptoms, while only the Functional Impairment subscale was associated with higher MDD symptoms. Moreover, engaging in collective action, but not individual action, significantly attenuated the association between CCA cognitive emotional impairment and MDD symptoms. Responses to open-ended questions asking about participants' worries and actions related to climate change indicated the severity of their worries and, for some, a perception of the insignificance of their actions relative to the enormity of climate change. These results further the field's understanding of CCA, both in general and specifically among emerging adults, and suggest the importance of creating opportunities for collective action to build sense of agency in addressing climate change.
\end{abstract}

Keywords Climate change $\cdot$ Anxiety $\cdot$ Depression $\cdot$ Activism $\cdot$ Agency $\cdot$ Emerging adults

\section{Introduction}

Increasing evidence indicates that climate change will have far-reaching impacts on human health, including mental health (Doherty \& Clayton, 2011; Hayes et al., 2018). A large body of literature has investigated the mental health consequences of one marker of climate change, weather-

Sarah E. O. Schwartz

seoschwartz@suffolk.edu

1 Department of Psychology, Suffolk University, Boston, MA, USA

2 Child Study Center, QUALab, Yale School of Medicine, New Haven, CT, USA

3 Inserm U1018, CESP, Team DevPsy, University Paris-Saclay, Villejuif, France

4 Maison de Solenn, Hospital Cochin AP-HP, Paris, France

5 Department of Psychology, College of Wooster, Wooster, OH, USA

6 Department of Social and Behavioral Sciences, Yale School of Public Health, New Haven, CT, USA related disasters, showing elevated rates of common psychiatric conditions like Major Depressive Disorder (MDD) and Generalized Anxiety Disorder (GAD) (Goldmann \& Galea, 2014; Hrabok et al., 2020), and a growing number of studies also suggest that rising temperatures can impair mental health and increase risk for suicidal behavior (Burke et al., 2018; Cianconi et al., 2020; Heo et al., 2021). However, a focus on weather-related disasters and temperature misses the full range of climate change exposures, including its subtler indicators, and their mental health consequences. Recent research indicates that even the existential threat of climate change may be associated with adverse mental 
health outcomes, particularly among younger populations, and recent studies have documented the existence of climate change anxiety (CCA). CCA is defined as negative responses associated with concerns about climate change (Clayton, 2020; Wullenkord et al., 2021). It may include cognitive, emotional, and behavioral responses, for example, persistent worries, psychological distress, or sleep difficulties related to long-term consequences of climate change, and can result in functional impairment, such as when concerns about climate change interfere with an individual's ability to engage fully in work, school, or relationships (Clayton \& Karazsia, 2020). This constellation of responses does not itself qualify as a psychiatric disorder, such as MDD or GAD, but indicates a source of stress that can have a negative impact on mental health (Clayton, 2020).

CCA has received growing attention in the popular press (Benoit et al., 2022), yet little research has examined how it relates to psychiatric symptoms, including symptoms of MDD and GAD. Furthermore, with more people, especially young people, taking action to address climate change, research is needed to explore how engaging in action may relate to both CCA and mental health symptoms.

\section{Climate Change Anxiety and Mental Health}

CCA is distinguished from responses that are associated with potentially traumatic climate-related experiences, such as extreme weather events, and is thought to arise from the fuller range of climate change-related exposures, including exposure to climate change via the media (Clayton, 2020). There is little consensus on how to conceptualize CCA from a mental health standpoint, with some describing it as a "pop-culture trend" (Grist, 2019), others as an appropriate and normal response, and still others as a clinical disorder that needs treatment (Taylor, 2020). Disagreement surrounding the construct may be in part due to criticism regarding medicalization of legitimate environmental concerns, but may also reflect the fact that, until recently, there were few measures of CCA, with existing measures focusing more generally on negative emotions related to climate change, rather than on CCA specifically (e.g., Eisenman et al., 2015; Searle \& Gow, 2010).

A recent measure developed by Clayton and Karazsia (2020) was designed to specifically emphasize clinically significant aspects of CCA. Factor analyses identified two subscales: cognitive emotional impairment, including rumination, difficulty sleeping or concentrating, and nightmares or crying; and functional impairment, reflecting difficulty engaging in work or academic responsibilities and relationships due to CCA. In the initial validation study of the CCA scale among adults in the United States using an MTurk sample, a measure of MDD and GAD symptoms was strongly correlated with cognitive emotional impairment $(r=.54)$ and functional impairment $(r=.47)$, indicating that CCA is related to, but not fully accounted by, depression and anxiety symptoms. While this provides important preliminary data about the relationship between CCA and psychiatric symptoms, the study used a brief four-item measure of combined MDD and GAD symptoms (PHQ-4; Kroenke et al., 2009a) without distinguishing between them. Similarly, another recent study showed an association between CCA and overall mental health among Filipino emerging adults (Reyes et al., 2021), but did not examine how CCA may relate differently to different types of psychiatric symptoms. Thus, there is a need for greater clarity on how CCA relates to the clinical constructs of MDD and GAD.

\section{Climate Change Anxiety and Climate Action}

Engaging in action in the face of climate change may be a way to regain power and build agency, which may, in turn, be associated with better mental health (e.g., Gong et al., 2011; Veronese et al., 2017). Research indicates that activism may protect against feelings of hopelessness in the context of overwhelming circumstances or when one's future wellbeing is in jeopardy (DeAngelo et al., 2016). Engaging in collective action can also result in feelings of empowerment and social support (Flanagan et al., 2007; Gorski, 2019) with some research indicating it may be associated with better long-term mental health (Boehnke \& Wong, 2011). At the same time, other research points to the psychological costs of activism, particularly activism related to personally-relevant issues (Chen \& Gorski, 2015). Engaging in climate activism typically means spending significant time thinking about climate change as well as devoting substantial psychological and/or material resources to a cause that, even in the best of circumstances, is likely to include extensive loss (Randall, 2015). This has the potential to leave activists feeling depleted and overwhelmed, contributing to anxiety and depression. Furthermore, when activism fails to achieve expected goals, this process may actually increase distress, anxiety, or despair (Albright \& Hurd, 2021; Kovan \& Dirkx, 2003).

Although there is limited research specific to the psychological effects of engaging in climate activism, qualitative research suggests that collective action may help climate activists manage their fears, foster hope, and increase feelings of connection (Kleres \& Wettergren, 2017; Nairn, 2019). Another qualitative study examining coping strategies among youth worried about climate change indicated that problem-focused coping strategies, including engaging in individual and collective action, were used by some youth to regulate climate change-related worries (Ojala, 2012). Finally, research has demonstrated associations between engaging in individual climate action, namely sustainable behavior, and happiness (Corral-Verdugo et al., 2015). 
Taken together, these studies point to the potential of climate action to reduce distress associated with the threat of climate change.

\section{Climate Change Anxiety and Activism Among Emerging Adults}

Young adults have been at the forefront of much of the recent climate-focused activism. This is consistent with historical trends in which young adults have often led major social change movements (Franklin, 2021). Reimer (2013) argued that young adults are the ideal target for engaging in environmental activism because of their developmental stage, environmental knowledge, and ability to be effective messengers. At the same time, young people's activism can also be stigmatized; for example, newspapers in the United States discussing climate change depict youth alternately as demanding activists, adultified kids, innocent victims, or ultimate saviors (Benoit et al., 2022).

Emerging adults may also be particularly affected by CCA. Research indicates that CCA and pessimism related to climate change are more commonly endorsed in adolescence and young adulthood when compared both to childhood (Ojala, 2012) and middle and late adulthood (Clayton \& Karazsia, 2020). In addition to elevated concerns about climate change specifically, emerging adults are more likely to meet criteria for a mental health disorder compared to other age groups (e.g., Henin \& Berman, 2016). MDD and GAD are particularly likely to develop in emerging adulthood (Kessler et al., 2005). Of note, college students appear to have been especially impacted by the COVID-19 pandemic, showing increased anxiety and distress relative to the general population (Wang et al., 2020). Thus, while young adults may be particularly well-positioned to engage in climate activism and are increasingly taking on the mantle of responsibility to address this global threat, it is also important to better understand how this may relate to their mental health, both for their sakes individually and for sustainability of the social movement.

\section{Current Study}

In the context of research indicating emerging adults' elevated levels of CCA and their leading role in climate-related action, this mixed methods study aimed to examine CCA, MDD and GAD symptoms and climate-related action within an emerging adult sample. Our primary aims were: 1) to examine associations between CCA and clinical measures of distress including GAD and MDD symptoms; 2) to investigate individual and collective climate action as potential moderators of associations between CCA and symptoms of GAD and MDD and 3) to explore the subjective, individualized experiences and behaviors of participants experiencing distress related to CCA. Aims 1 and 2 were investigated quantitatively, while Aim 3 was addressed by drawing on open-ended responses to prompts about participants' worries and actions related to climate change, focusing on a subsample of participants reporting elevated levels of CCA and probable GAD and/or MDD diagnoses based on their quantitative data. Due to the limited research on climate change anxiety and mixed evidence related to the role of activism in ameliorating or exacerbating distress, no specific hypotheses were warranted.

\section{Method}

\section{Participants and Procedures}

Participants included undergraduate and graduate students at universities in the United States, ages 18-35 years old. Participants were recruited to complete an anonymous crosssectional web-based survey between October and December 2020. Of note, this data collection occurred in the context of the COVID-19 pandemic during which most universities were engaged in remote learning. This was also a time period of heightened political anxiety in the United States in the context of the November 2020 presidential election (Canady, 2019). Participants were recruited both from the psychology participant pool at an urban, private university in the Northeast, as well as via email outreach and social media more broadly. Participants were given the option to enter a raffle to receive one of three $\$ 100$ electronic gift cards or, for those students within the participant pool, research credit in their psychology course.

A total of 323 participants completed the survey; of these $284(88.0 \%)$ provided complete data on all variables in the current study and comprised the analytic sample. Among them, 223 (78.5\%) participants provided free-response comments that have been included in a qualitative analysis.

\section{Measures}

Climate Change Anxiety was assessed using the Climate Change Anxiety Scale (Clayton \& Karazsia, 2020). This 13-item scale assesses the negative cognitive and emotional impacts and functional impairment related to perceptions of climate change. It includes two subscales, including Cognitive Emotional Impairment (8 items) and Functional Impairment (5 items). Items in the Cognitive Emotional Impairment subscale include, "I go away by myself and think about why I feel this way about climate change," while the Functional Impairment subscale includes items such as, "My concerns about climate change interfere with my ability to get work or school assignments done." Responses were reported using a Likert scale, with response options 
ranging from $1=$ Never to $5=$ Almost always. Mean scores were calculated, with higher scores indicating higher levels of climate anxiety. This scale showed good reliability and validity in a U.S. adult sample and in a Filipino emerging adult sample (Clayton \& Karazsia, 2020; Reyes et al., 2021). Reliability in the current sample was acceptable (total score $\alpha=.89$; Cognitive Emotional Impairment subscale $\alpha=.87$; Functional Impairment subscale $\alpha=.79$ ).

Individual Climate Action was measured using a fiveitem scale to assess sustainable behavior used in the original validation study of the Climate Change Anxiety scale (Clayton \& Karazsia, 2020). Sample items included, "I recycle" and "I feel guilty if I waste energy." This scale was developed with the Climate Change Anxiety scale and was based loosely on the Drive for Muscularity Scale, with the goal of looking at behavior that was motivated by anxiety or emotions (McCreary \& Sasse, 2000). Factor analysis distinguished this scale from the anxiety related items in the Climate Change Anxiety scale, and it was labeled Behavioral Engagement. Since items focused only on individual behavior to address climate change, in the current study we refer to this scale as Individual Climate Action to differentiate it from the collective actions measured in the Climate Activism scale. Responses were reported using a Likert scale, with response options ranging from $1=$ Disagree completely to $5=$ Agree completely. Mean scores were calculated, with higher scores indicating higher levels of engagement in individual climate action. Reliability in the current sample was acceptable $(\alpha=.68)$.

Climate Activism was measured using an adapted version of the Environmental Actions Scale (Alisat \& Riemer, 2015). This 18 -item scale assesses how frequently participants engaged in a range of environmental actions including both participating in civic action (e.g., "Took part in a protest/rally about an environmental issue"), and leading civic action (e.g., "Organized a petition [including online petitions] for an environmental cause)." We adapted the scale such that participants responded in reference to the prior year (rather than the prior 6 months) and were instructed to respond based on their behaviors prior to the COVID19 pandemic, since some forms of action were no longer possible at the time of data collection due to pandemicrelated social distancing measures. Responses were reported using a Likert scale, with response options ranging from $0=$ Never to $4=$ Frequently. Mean scores were calculated, with higher scores indicating higher levels of engagement in climate activism. This scale has been used with college student populations internationally and has shown good reliability and validity (Alisat \& Riemer, 2015). Reliability in the current sample was good $(\alpha=.93)$.

Major Depression Disorder symptoms were measured using the eight-item Patient Health Questionnaire (PHQ-8; Kroenke et al., 2009b). The PHQ-8 assesses eight of the nine criteria for MDD in the Diagnostic and Statistical Manual for Mental Disorders, Fourth Edition (DSM-IV), excluding symptoms of suicidality (American Psychiatric Association, 1994). Participants were asked to report how often during the past two weeks they were bothered by each of the eight symptoms described. Response options ranged from $0=$ Not at all to $3=$ Nearly every day. Sum scores were calculated, with higher scores indicating greater severity of MDD symptoms, and scores of 10 or above considered indicative of a probable MDD diagnosis (Kroenke et al., 2009b). Reliability in the current sample was good (total score $\alpha=.88$ ).

Generalized Anxiety Disorder symptoms were measured using the Patient Health Questionnaire Generalized Anxiety Disorder Scale (GAD-7; Spitzer et al., 2006). The GAD-7 assesses anxiety symptoms based on the criteria for GAD used in the Diagnostic and Statistical Manual for Mental Disorders, Fourth Edition (DSM-IV; American Psychiatric Association, 1994). Participants were asked to report how often during the past two weeks they were bothered by each of the seven symptoms described. Response options ranged from $0=$ Not at all to $3=$ Nearly every day. Sum scores were calculated, with higher scores indicating greater severity of GAD symptoms, and scores of 10 or greater indicative of probable GAD (Spitzer et al., 2006). Reliability in the current sample was good (total score $\alpha=.90$ ).

Climate Change Experience. This three-item scale was included as a covariate both to be consistent with prior research on this topic (Clayton \& Karazsia, 2020) and to facilitate examination of associations between CCA and mental health symptoms above and beyond participants' direct climate change exposure. The scale assessed the extent to which individuals have experienced the impacts of climate change (e.g., "I have been directly affected by climate change"; Clayton \& Karazsia, 2020). Responses were reported using a Likert scale, with response options ranging from $1=$ Disagree completely to $5=$ Agree completely. Mean scores were calculated, with higher scores indicating greater experience with the impacts of climate change. Reliability was acceptable $(\alpha=.79)$.

Demographics. Demographic covariates were selected based on prior research showing their associations with climate change anxiety, environmental activism, or MDD and GAD symptoms (Clayton \& Karazsia, 2020; Kessler et al., 2005). Age was included as a continuous variable. Gender identity was a three-level categorical variable (man; woman; transgender, non-binary, or other). Given the small number in the latter category $(n=6)$, this was dichotomized into male $=1$, all others $=0$, based on prior research showing those who identify as women, transgender, non-binary, and other gender reporting significantly higher MDD and GAD symptoms than those who identify as men (Kessler et al., 2005; Reisner et al., 2016). Participants reported their race using a single item allowing for multiple responses. The 
following dummy codes were then derived: White; Black; Asian; Latinx or Hispanic; and Other (including Native American/Alaska Native, Middle Eastern/North African, Multiracial, and "not listed"). Although these categories were not mutually exclusive, White was used as a reference group to facilitate model convergence. Dummy codes were also included for whether the participant was a financial aid recipient; a first-generation college student; born in the United States; and a graduate (versus undergraduate) student.

Open-ended questions were included at the end of the survey including: 1) "What, if anything, worries you most about climate change?" and 2) "What, if anything, do you do on a regular basis to address climate change?" We used written open-ended accounts to allow participants to take their time, reflect independently without researcher presence, and focus on experiences most salient to them. Anonymity also may reduce social desirability and facilitate candor (Braun et al., 2020).

\section{Data Analysis}

Quantitative analyses were conducted in SPSS version 27 (IBM Corp, 2020) and consisted of three steps. First, a series of preliminary analyses were conducted, including computation of descriptive statistics for all variables in the analysis and a correlation matrix. Independent-samples $t$-tests and chi-square analysis were used to compare the 284 participants in the analytic sample and the 39 participants who were dropped due to missing data. Second, two multivariable linear regression models were run to examine the CCA subscales and individual action and climate activism as predictors of MDD and GAD symptoms, respectively, controlling for demographic covariates and direct climate change experience. Third, we examined individual climate action and climate activism as moderators of the two CCA subscales using Model 1 of the PROCESS macro (Hayes, 2017). A total of eight moderation models were run, four for MDD symptoms and four for GAD symptoms, and each with one interaction term. Variables comprising interaction terms were mean-centered, and interactions were then computed as their product. Centered variables, interactions, and covariates were entered as predictors of the dependent variable. In cases of significant interaction terms, the conditional effects of CCA at low (1 standard deviation $[S D]$ below the mean), mean, and high (1 SD above the mean) levels of the moderator were recorded, and predicted values were plotted in Microsoft Excel.

Qualitative analysis was informed by the COREQ checklist for quality and rigor in qualitative research (COREQ). The analysis was exploratory, and so we adopted an inductive approach focusing on what was meaningful to participants about climate change rather than exploring their accounts through a specific theoretical lens. We used reflexive thematic analysis to develop rich experiential themes that could capture latent features of the data and offer nuanced insights into participants' subjective experiences. Following Braun and Clarke's (2006) six-stage process, we familiarized ourselves with the dataset, and then developed initial codes and themes, using half the accounts. We reviewed these themes against the data, and then analyzed the remaining half of the open comments, reviewing and developing themes throughout. All findings presented below provide theoretical sufficiency (i.e., reaching a sufficient depth of understanding for the researcher to build a theory, and have been reviewed against the data to ensure their redundancy (i.e., that they reflect the views of several participants). One author (LB) undertook analysis and engaged in regular debriefing with others in the research group (SS, SC, SL).

To better understand the concerns of those experiencing higher levels of distress, we also conducted an in-depth analysis of a subsample of participants reporting elevated CCA and mental health distress based on the quantitative survey data. Specifically, a "high distress" category was created for participants whose mean CCA scores were greater than 2 (more than "rarely") and who were classified as having either probable MDD and/or GAD based on their survey responses.

\section{Results}

\section{Preliminary Analyses}

Table 1 lists descriptive statistics for all variables in the analysis. Participants were 23.17 years old on average $(S D=3.87)$; the majority identified as women $(78.9 \%)$ and White (66.2\%). More than half (53.5\%) were graduate students, and $68.0 \%$ were financial aid recipients. Based on participants' scores on the PHQ-8 and GAD-7, 28.9\% were classified as having probable MDD and $37.0 \%$ as having probable GAD. The CCA cognitive emotional impairment mean was $1.41(\mathrm{SD}=.54$; range: $1.00-4.00)$, and the CCA functional impairment mean was $1.67(\mathrm{SD}=.57$; range: 1.00-3.80), both representing average responses between "Never" and "Rarely."

Table 2 shows a correlation matrix for all variables in the analysis. Of note, both CCA subscales were positively and significantly associated with MDD and GAD symptoms (both $p<.001$ ). Individual climate action was positively and significantly associated with the CCA functional impairment subscale $(p<.01)$, but not the CCA cognitive emotional impairment subscale $(p=.396)$. In contrast, climate activism was significantly and positively associated with both CCA subscales (both $p$ 's <.001). Neither individual climate actions nor climate activism were significantly associated 
Table 1 Descriptive statistics for analytic sample

\begin{tabular}{|c|c|c|}
\hline & $\mathrm{M}(\mathrm{SD})$ & $\mathrm{n}(\%)$ \\
\hline \multicolumn{3}{|l|}{ Demographic characteristics } \\
\hline Age (years) & $23.17(3.87)$ & \\
\hline \multicolumn{3}{|l|}{ Gender identity } \\
\hline Man & & $54(19.0 \%)$ \\
\hline Woman & & $224(78.9 \%)$ \\
\hline Transgender, non-binary, or & & $6(2.1 \%)$ \\
\hline \multicolumn{3}{|l|}{ 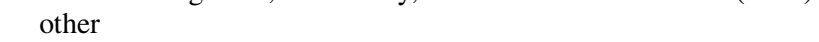 } \\
\hline \multicolumn{3}{|l|}{ Race and ethnicity } \\
\hline White & & $188(66.2 \%)$ \\
\hline Black & & $15(5.3 \%)$ \\
\hline Asian & & $53(18.7 \%)$ \\
\hline Hispanic or Latinx & & $34(12.0 \%)$ \\
\hline Other & & $24(8.5 \%)$ \\
\hline Financial aid recipient & & $193(68.0 \%)$ \\
\hline First-generation college student & & $62(21.8 \%)$ \\
\hline U.S. born & & $241(84.9 \%)$ \\
\hline \multicolumn{3}{|l|}{ Student status } \\
\hline Undergraduate & & $132(46.5 \%)$ \\
\hline Graduate & & $152(53.5 \%)$ \\
\hline Climate change experience & $3.47(1.02)$ & \\
\hline \multicolumn{3}{|l|}{ Climate change anxiety } \\
\hline Cognitive emotional impairment & $1.41(0.54)$ & \\
\hline Functional impairment & $1.69(0.57)$ & \\
\hline Individual climate action & $4.19(0.56)$ & \\
\hline Climate activism & $1.30(0.83)$ & \\
\hline \multicolumn{3}{|l|}{ Mental health } \\
\hline MDD symptom severity & $7.20(5.47)$ & \\
\hline Probable MDD diagnosis & & $82(28.9 \%)$ \\
\hline GAD symptoms & $8.27(5.43)$ & \\
\hline Probable GAD diagnosis & & $105(37.0 \%)$ \\
\hline
\end{tabular}

$\mathrm{GAD}=$ Generalized Anxiety Disorder. MDD $=$ Major Depressive Disorder. Categories for race and ethnicity were not mutually exclusive

with MDD or GAD symptoms (all $p$ 's > .05). Lastly, higher individual climate action was significantly associated with higher climate activism $(p<.001)$.

No significant differences on any of the variables in the current study were detected between the 284 participants in the analytic sample and the 39 participants dropped due to missing data (all $p$ 's $>.05$ ).

\section{Major Depressive Disorder Symptoms}

The results of the multivariate linear regression model predicting MDD symptoms are shown in Table 3. The model was statistically significant, $F(15,268)=3.71, p<.001$, accounting for $17.2 \%$ of the variance in MDD symptoms. Higher CCA functional impairment was significantly associated with higher MDD symptoms $(p=.026)$, whereas the association between CCA cognitive emotional impairment and MDD symptoms was non-significant $(p=.131)$. Higher individual climate action was marginally associated with lower MDD symptoms $(p=.071)$, whereas the relationship between climate activism and MDD symptoms was nonsignificant $(p=.527)$.

The addition of the interaction between CCA cognitive emotional impairment and climate activism to the model was statistically significant, $F(1,267)=4.39, p=.037$, and accounted for $1.3 \%$ additional variance in MDD symptoms. Analysis of conditional effects showed that CCA cognitive emotional impairment was significantly associated with MDD symptoms for participants reporting low and mean levels of climate activism $(B=3.33, S E=1.29, p=.010$, and $B=2.12, S E=0.90, p=.020$ ), whereas the relationships was non-significant for those reporting high environmental activ$\operatorname{ism}(B=0.90, S E=0.80, p=.261)$. Figure 1 further shows that climate activism buffered the impact of CCA cognitive emotional impairment on MDD symptoms. The interaction between CCA functional impairment and climate activism was non-significant, $F(1,267)=0.33, \Delta R^{2}=.001, p=.566$, as were the interactions between both CCA subscales and individual climate action (cognitive emotional impairment: $F[1,267]<0.01, \Delta R^{2}<.001, p=.960$; functional impairment: $\left.F[1,267]=0.05, \Delta R^{2}<.001, p=.821\right)$.

\section{Generalized Anxiety Disorder Symptoms}

Table 3 also shows the results of the multivariable linear regression model predicting GAD symptoms. The model was significant, $F(15,268)=5.37, p<.001$, accounting for $23.1 \%$ of the variance in GAD symptoms. Higher levels of both CCA cognitive emotional impairment and CCA functional impairment were significantly associated with higher GAD symptoms ( $p=.046$ and $p=.011$, respectively). Higher individual action was associated with lower GAD symptoms $(p=.042)$, whereas the relationship between climate activism and GAD symptoms was non-significant $(p=.396)$.

Neither the interaction between CCA cognitive emotional impairment and climate activism, nor the interaction between CCA functional impairment and climate activism accounted for additional variance in GAD symptoms ( $F[1$, $267]=1.00, \Delta R^{2}=.003, p=.317$, and $F[1,267]=1.93, \Delta$ $R^{2}=.006, p=.166$, respectively). Interactions between CCA subscales and individual climate action were also non-significant (cognitive emotional impairment: $F[1,267]<0.01, \Delta$ $R^{2}<.001, p=.960$; functional impairment: $F[1,267]=0.03$, $\left.\Delta R^{2}<.001, p=.861\right)$. 


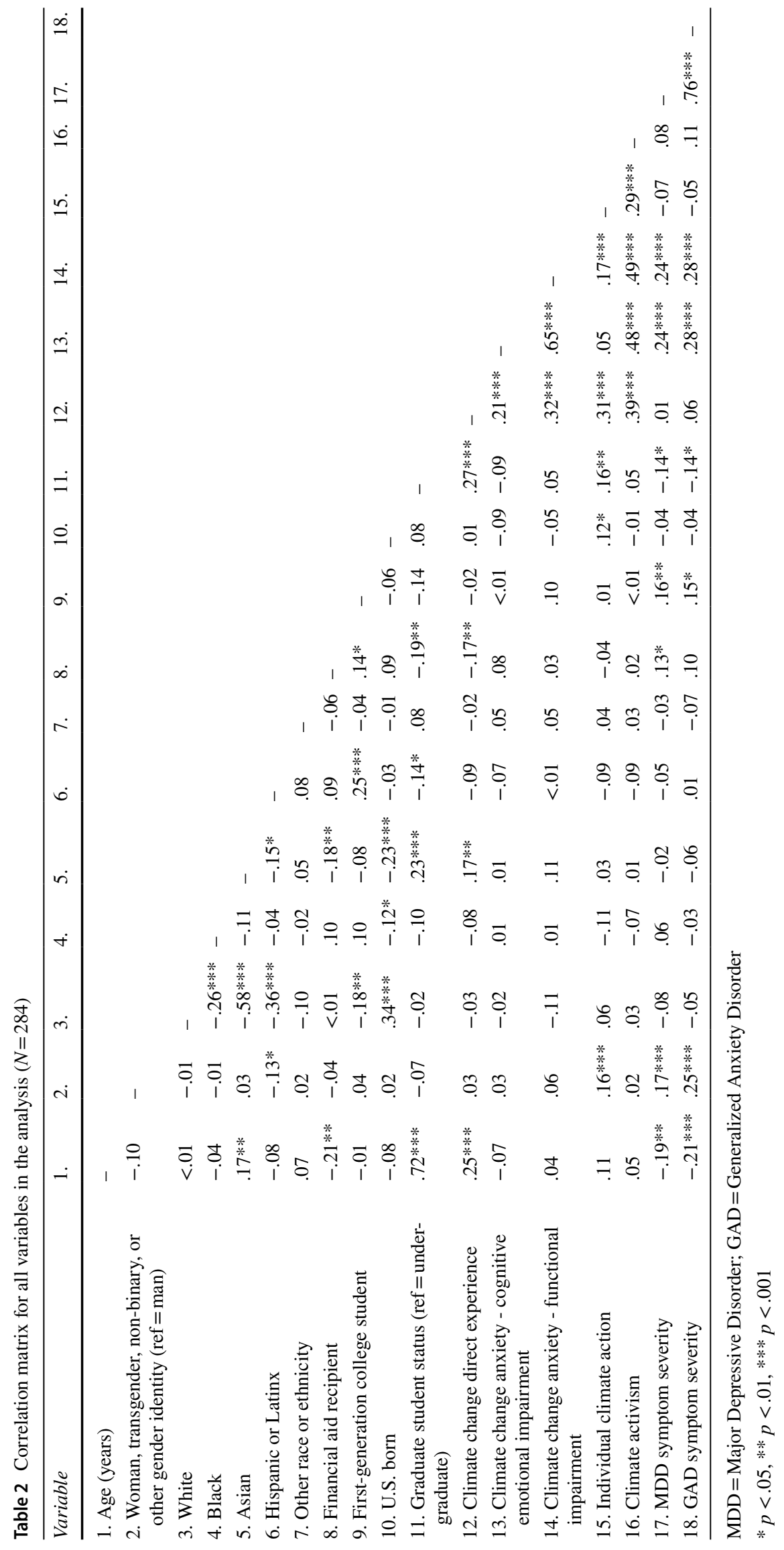


Table 3 Results of multivariable regression analyses predicting mental health outcomes

\begin{tabular}{|c|c|c|}
\hline & $\begin{array}{l}\text { MDD symptoms } \\
B(S E)\end{array}$ & $\begin{array}{l}\text { GAD symptoms } \\
B(S E)\end{array}$ \\
\hline Constant & $13.34(3.66)^{* * *}$ & $14.82(3.50)^{* * *}$ \\
\hline \multicolumn{3}{|l|}{ Demographic characteristics } \\
\hline Age (years) & $-0.28(0.12)^{*}$ & $-0.31(0.11)^{* *}$ \\
\hline \multicolumn{3}{|l|}{ Gender identity } \\
\hline Man & $-2.09(0.80)^{*}$ & $-3.28(0.77)^{* * *}$ \\
\hline $\begin{array}{l}\text { Woman, transgender, } \\
\text { non-binary, or other (ref) }\end{array}$ & - & - \\
\hline \multicolumn{3}{|l|}{ Race } \\
\hline White (ref) & - & - \\
\hline Black & $0.09(1.41)$ & $-1.81(1.35)$ \\
\hline Asian & $-0.18(0.86)$ & $-1.01(0.82)$ \\
\hline Latinx or Hispanic & $-1.56(1.01)$ & $-0.18(0.97)$ \\
\hline Other & $-0.22(1.11)$ & $-1.32(1.06)$ \\
\hline Financial aid recipient & $0.89(0.69)$ & $0.61(0.66)$ \\
\hline $\begin{array}{l}\text { First-generation college } \\
\text { student }\end{array}$ & $1.99(0.79)^{*}$ & $1.63(0.76)^{*}$ \\
\hline U.S. born & $-0.57(0.92)$ & $-0.85(0.88)$ \\
\hline \multicolumn{3}{|l|}{ Student status } \\
\hline Undergraduate & $0.58(0.93)$ & $0.93(0.89)$ \\
\hline Graduate (ref) & - & \\
\hline Climate change experience & $0.11(0.36)$ & $0.37(0.34)$ \\
\hline \multicolumn{3}{|l|}{ Climate change anxiety } \\
\hline $\begin{array}{l}\text { Cognitive emotional } \\
\text { impairment }\end{array}$ & $1.20(0.80)$ & $1.53(0.76)^{*}$ \\
\hline Functional impairment & $1.69(0.75)^{*}$ & $1.85(0.72)^{*}$ \\
\hline Individual climate action & $-1.08(0.60)$ & $-1.17(0.57)^{*}$ \\
\hline Climate activism & $-0.29(0.46)$ & $-0.38(0.44)$ \\
\hline
\end{tabular}

$\mathrm{GAD}=$ Generalized Anxiety Disorder MDD $=$ Major Depressive Dis order

$* p<.05, * * p<.01, * * * p<.001$

\section{Qualitative Findings}

\section{Summary of General Qualitative Findings}

From the full sample of 284 participants, 223 participants (79.9\%) responded to the optional open-ended question asking about worries surrounding climate change, and 211 (75.7\%) responded to the optional open-ended question asking them to describe any actions they took to address climate change. No one wrote about actions to address climate change without describing worries first. Responses about worries varied from 1 to 316 words $(M=39.02)$, and those about actions varied from 1 to 227 words $(M=30.64)$.

Table 4 includes a summary of the general themes identified in participants' responses. Responses describing worries about climate change illustrated four major and recurrent themes: environmental damage, collective inaction, human global suffering, and individual suffering. Responses describing actions to address climate change showed that almost all participants who responded to the question engaged in individual actions that were measured in the Individual Action scale (e.g., recycling, turning off lights and saving energy). Many also detailed individual actions that were not assessed in the scale. Collective actions were less commonly described than individual actions, and all the collective actions described by the participants were included in the Climate Activism scale.

\section{Qualitative Analysis of High Distress Subsample}

Among 47 participants with elevated CCA and probable MDD and/or GAD, 37 (78.7\%) responded to the optional open-ended questions. In contrast to the general qualitative findings, this high distress subsample of 37 comments particularly emphasized 5 distinctive themes: (1) the deadly
Fig. 1 Association between CCA cognitive emotional impairment and MDD symptoms for participants with low and high levels of climate activism

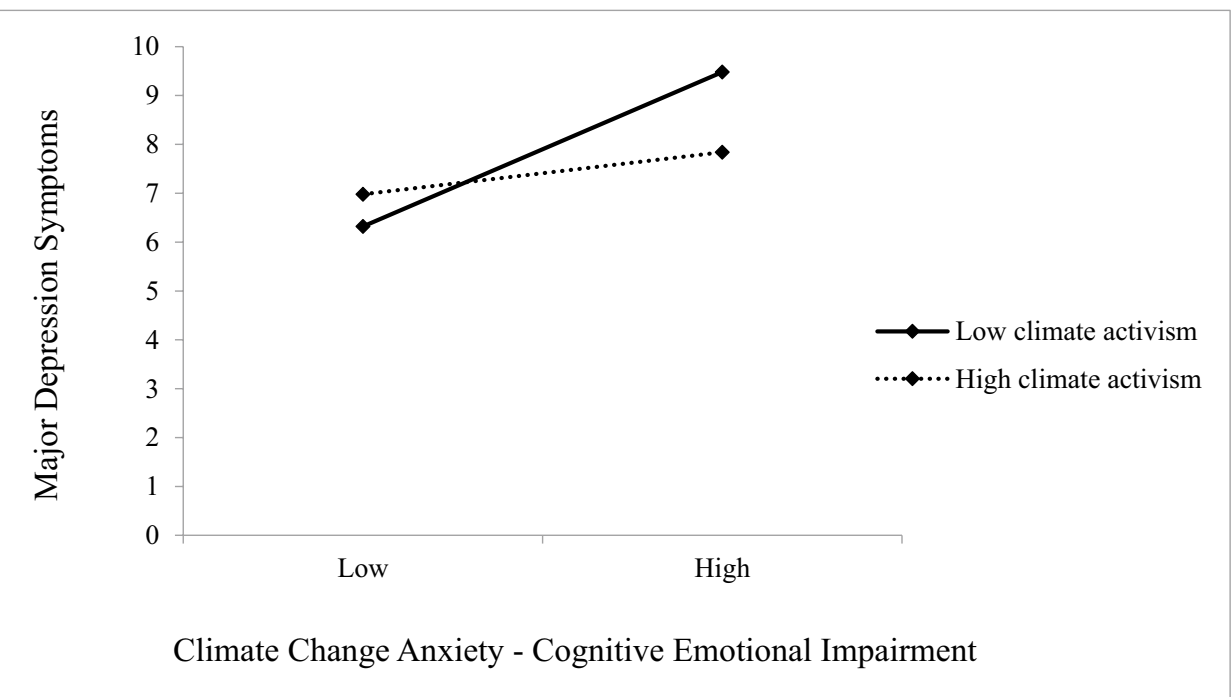


Table 4 Summary of general qualitative findings

Main worries about climate change

Environmental damage

Collective inaction

Human suffering

Individual suffering

Actions to address climate change

Individual actions mentioned in existing scale

Individual actions not mentioned in existing scale

Collective actions
Global collapse; Loss of biodiversity; Loss of places; Unpredictable weather events

The barrier of US lifestyle; Governments' inaction (US and international); The insignificant impact of individual actions; Capitalism, Corruption; Lack of education, climate denial; Politicization and manipulation of public opinion; Indifference to existing solutions; Environmental solutions that create new problems

Disasters, starvation, health problems; Chained events: from migrations to wars; Lack of climate justice and social justice

Relatives or one's generation being harmed; Children, grandchildren and future generations being harmed; Considering the decision of not having children

Recycling; Saving energy; Turning off lights

Reducing consumption of goods and production of trash, compost; Avoiding disposable, single use material, and packaging; Buying second-hand products, repairing; Buying from small, local, and sustainable businesses; Diet (reducing meat consumption, vegetarian, entirely plant-based); Producing (vegetables, herbs, skincare products); Avoiding online purchases; Transportation (walking, cycling, using public transportation, carpooling, using an electric or hybrid car, avoiding planes); Choosing a professional field to address climate change; Saving to afford climate conscious purchases (insulation, electric car, geothermal or solar energy)

Educating oneself about environmental science; Spreading awareness (online, sending emails to elected officials, talking to other people about climate change); Financial support to an environmental cause; Joining or leading advocacy groups, clubs, and student groups; Organizing events, supporting local initiatives; Supporting politicians who have introduced climate change policies and talking to friends about them; Advocating for alternative energy (i.e. nuclear power as a stop gap measure); Getting involved in research groups focused on climate change; Teaching other students about climate change threat posed by climate change, (2) its immediacy, celerity, and irreversibility, (3) the future global chaos due to climate change, (4) their helplessness in face of collective inaction, and (5) their paralysis and fewer collective actions.

The Deadly Threat Posed by Climate Change Respondents gave numerous accounts of the "existential threat" posed by climate change for humans (" $[\mathrm{me}]$ and my peers' future, [...] marginalized populations"), and for animals ("Certain animals [are] dying including polar bears. I am a big animal lover"). Several participants were afraid of massive yet undefined threats. One participant wrote, "I'm worried about the worst that could happen in my lifetime," with the planet becoming "unlivable in a few decades" due to the combination of scarce resources and overpopulation, while another wrote, "Many more animals and insects we need to survive as a population will go extinct. I am more afraid of running out of natural materials because of overpopulation as well." Many participants described deadly threats of "widespread famine," and climate change-related disasters such as "fires and natural storms" that were expected to "wipe out populations." Others tackled the responsibility of capitalism in what they compared to the murder of millions of persons: "larger corporations [...] will kill us all."
The Immediacy, Celerity and Irreversibility of Climate Change In contrast with the overall sample, the high distress subsample stressed the immediacy of climate change and already witnessing its effects, stating "we already know a lot of what will happen and are experiencing it." Their frequent use of the present tense emphasized already current consequences, for example, "My boyfriend's family [...] receive small earthquakes all the time now and big ones often starting only recently." The celerity of climate change was also stressed by these participants, who envisioned disaster occurring within a narrow time frame (e.g., "what the world will look like in 10-20 years"). Their awareness of a countdown was distressful ("something that really scared me was the clock in New York. It is scary knowing we have such little time until we see catastrophe") and led them to question the responsibility of bringing "kids into a world that is currently on a climate clock." The irreversibility of an upcoming "permanent damage" was stressed by the participants. Not only did some participants refer to irreversible changes (e.g., "we will no longer be able to see the world as it is"), but others also went so far as to describe the world as "dying at a rapid rate."

Chained Events Leading to Global chaos This subsample of participants expected the impact of climate change to "exacerbate the problems that many people already worry about" 
and provoke chained-events leading to "future pandemics. Future economic collapse." One participant noted, "Almost everything will be affected by climate change, mostly negatively." Participants worried about "the civil unrest caused by the migration" of "millions of people," that will exacerbate "ethnic/racial and [socioeconomic] inequality." Thus, they expected climate change to provoke conflict escalation, describing a process in which "Weather becomes less reliable, people move around, life becomes more precarious for many, perhaps fervor in group identification increases (e.g., religious), and the chances for conflict to spark increase," including "the chances of nuclear weapon usage."

Helplessness in Face of Collective Inaction Participants in the high distress subsample lamented decades of collective inaction in a similar way as the overall population of the survey, stating, "We've known this was coming for 50 years, and there is no real commitment to reduce the impact of oil and pollution from large corporations." Citizens' indifference, ignorance, and mistrust was worrisome for these participants as well as the politicization of the issue. Just as the overall population of the survey, they stressed the lack of "large, structural changes," supported by the interests of the few wealthiest actors, such as "corporations", "states" and "top billionaires." The selfishness and impunity of such attitudes was stressed by the participants, noting, "[they] tend to not care because it doesn't affect them," "no one is holding them to account" and "a fraction of their wealth would be sufficient to almost stop climate change."

While similar themes of helplessness were noted in both the general sample and the high distress subsample, those in the subsample expressed their helplessness more vividly than the others. While they described diverse individual actions consistent with the general results most of them started their accounts by humble regrets, "I try," "I attempt," or "I wish I could." Their awareness of the almost insignificant impact of individual actions appeared burdening. For example, one participant stated, "It is not bad at all to make environmentally friendly choices on an individual level, but there are much larger powers at play that are actively harming the climate. Without directly taking action to stop or regulate that harm on a larger scale, individual lifestyle changes will not make a large enough impact to save us."

Paralysis and Fewer Collective Actions While the 37 responses about worries in this subsample tended to be more detailed and longer than those of the general population, the rare concise answers conveyed a desperate tone. To the question "what worries you about climate change?" a few participants simply wrote: "Everything." This high distress subsample described climate change disrupting their engagement with goals typically salient in emerging adulthood such as education, career, and family-related goals. One participant stated, "Even going to college and thinking of my future at times feels misguided and naive when facing the reality that there is a large chance that I won't be able to have children, a future or a stable career in a world that is devastated by climate change." Sacrificing parenthood to reduce population growth appeared to be one way some participants strived to maintain agency over what remained in their control, noting "Myself as a privileged person has a choice, is it responsible to bring more people into the world when I know population growth is a driver of climate change?" While these comments focused on disengaging from typical goals and milestones that may provide a sense of purpose or meaning, there was no mention of these being replaced with other goals or purpose. Actions that participants in this subsample were taking to address climate change primarily emphasized individual actions to reduce their carbon footprint, and those that did mention collective actions provided fewer details about those actions than the overall population. These participants seemed motivated to take action, but unable to engage in meaningful collective action. One participant described, "It is scary knowing we have such little time until we see catastrophe. I wish I could do more to help such as joining a club and being active in doing this but because of the pandemic it is just very hard to go out and meet with people [...] I wish there was a climate change group or something that I can talk to more people about it." Table 5 provides sample paired responses to the questions about worries and actions in the high distress subsample and highlights the immensity of their worries along

Table 5 Examples of worries and actions for high distress subsample $(n=37)$

\begin{tabular}{lll}
\hline Participant & What if anything worries you about climate change? & What if anything do you do to address climate change? \\
\hline A & Actually, everything. & Taking action in petitions. \\
B & The world imploding & Recycling \\
C & The way that people of the world, private corporations, and elected & rant about capitalism killing us all online. oh, and \\
& governments reacted to covid-19 proves to me we are absolutely & drinking oat milk instead of dairy \\
& $\begin{array}{l}\text { beyond fucked with climate-change. Like there is literally no hope that } \\
\text { millions (or billions) more people won't die by climate-change-related }\end{array}$ & \\
& causes and people/corporations/governments still won't take the neces- & \\
& sary action. & \\
\end{tabular}


with a perception of the insignificance or even absurdity of their actions in the face of climate change.

\section{Discussion}

The results of this study extend previous research on CCA to examine associations that distinguish between clinical measures of GAD and MDD. While both the CCA functional impairment and cognitive emotional impairment subscales were significantly associated with GAD symptoms, only CCA functional impairment was associated with higher MDD symptoms. Climate activism moderated the association between CCA cognitive emotional impairment and MDD symptoms, with a significant association between CCA and MDD emerging for those with low levels of activism, but no significant association for those with high levels of activism. Additionally, engagement in individual action was significantly associated with lower levels of GAD symptoms, but no moderation effects were detected for individual action. Qualitative data provided a deeper understanding of emerging adults' worries and actions, indicating the severity of worries related to climate change among the majority of participants but particularly striking among those with higher levels of CCA and mental health distress.

Interestingly, although the fears and worries described even within the full sample were significant, mean levels of CCA were relatively low, in fact, lower than the levels reported in the MTurk sample surveyed in the original measure validation study: for cognitive emotional impairment $1.41(\mathrm{SD}=.54)$ in the current sample compared to 1.75 $(\mathrm{SD}=.97)$ and for functional impairment $1.67(\mathrm{SD}=.57)$ in the current sample compared to 2.09 ( $\mathrm{SD}=1.08$; Clayton \& Karazsia, 2020). While this is surprising in that the original study showed that young adults had higher levels of climate anxiety than middle or older adults, it is likely that the current sample of undergraduate and graduate students reflected generally high-functioning individuals. Thus, an understanding of the severity of climate change does not necessarily impair functioning. At the same time, the majority of our sample reported having some experience with symptoms of climate change anxiety (i.e., reporting experiencing some symptoms at least "rarely"), suggesting the widespread impact of CCA.

These results help build on our understanding of CCA as a construct and how it relates to clinical measures. Previous research has shown that CCA is positively correlated with a combined measures of MDD and GAD symptoms (Clayton \& Karazsia, 2020) and with a general measure of mental health (Reyes et al., 2021). The current study demonstrated that both cognitive emotional and functional impairment CCA subscales were significantly associated with GAD symptoms, but only CCA functional impairment was associated with higher MDD symptoms, suggesting that although there can be overlap in some functional impairment symptoms in CCA and MDD, the cognitive and emotional components are distinct. These results are consistent with a recent study examining feelings of anxiety, depression, and anger related to climate change that showed that feelings of anxiety related to climate change were associated with anxiety and stress symptoms, but not depression symptoms (Stanley et al., 2021). More generally, associations between CCA subscales and both GAD and MDD scales were not large, suggesting that CCA should not be viewed simply as a manifestation of GAD or MDD, nor as a clinical disorder.

While our results indicate that CCA is distinct from GAD and MDD, the experience of CCA may contribute to or coexist with GAD or MDD. Qualitative data from those reporting higher levels of CCA and mental health symptoms demonstrated the way in which CCA may manifest among emerging adults who are also experiencing clinical symptoms, namely in diffuse and acute worries, feelings of helplessness, and an overall sense of hopelessness and doom. Moreover, responses demonstrated how climate change anxiety can pose a barrier to engaging with goals typically salient in emerging adulthood such as education, career, and family-related goals, which may contribute to a loss of meaning or purpose. This may be of particular concern in the context of an emerging adult population that is already more vulnerable to mental health distress.

Engaging in collective action, however, may have some capacity to protect against depression for emerging adults experiencing climate anxiety. This is consistent with research and theory on the role of agency in supporting the mental health of vulnerable populations (Gong et al., 2011; Veronese et al., 2017). Engaging in collective action may combat feelings of despair and helplessness and foster feelings of hope. Collective action also brings with it community connection and social support, which supports health and well-being (e.g., Lorimer et al., 2020; Velez \& Moradi, 2016). These results provide initial quantitative support for previous qualitative research in which climate activists describe collective action as a way to manage their fears, build hope, and create feelings of connection (Kleres \& Wettergren, 2017; Nairn, 2019). Participating in collective action may also serve similar functions as behavioral activation, an evidence-based treatment for MDD, and contribute to decreasing MDD symptoms.

Whereas individual action was associated with lower levels of GAD, it did not show the same capacity to buffer associations between CCA and mental health as collective action, suggesting the unique role of collective action in the face of overwhelming circumstances. This could be in part due to limitations of the scale itself, since qualitative responses indicated a much broader repertoire of individual actions that participants were engaging in than those represented 
in the scale. At the same time, qualitative responses also reflected perceptions of the insignificance of individual action in the face of the immensity of the problems posed by climate change. Nevertheless, the majority of our sample reported engaging in individual actions to address climate change, and it is encouraging that these actions may be associated with lower levels of GAD.

Quantitative and qualitative results suggest that CCA is impacting emerging adults broadly. While experiencing CCA in of itself does not constitute a clinical disorder, the experience of CCA may contribute to clinical disorders and, simply given the prevalence of both CCA and mental health disorders among emerging adults, they are likely to co-occur. In such cases, clinical intervention may be merited, and it is important for mental health professionals to have sufficient climate literacy to be able to effectively address CCA in therapeutic settings (Clayton et al., 2021). For example, it is important that therapists acknowledge that even severe worries about climate change are legitimate and normative among emerging adults, rather than viewing them solely as a symptom of a disorder. Existential therapies and thirdwave cognitive-behavioral approaches focusing on meaning, values, and acceptance may be an especially good fit for emerging adults experiencing CCA and seeking treatment. Additionally, proponents of ecotherapy have suggested that developing an environmental identity and engaging in environmental conservation may be an effective approach to treating climate anxiety (Doherty, 2016; Palinkas et al., 2020). More broadly, whether in the context of formal treatment or community, work, and educational contexts, creating opportunities to engage in collective action and, by doing so, build agency and connection, may be particularly beneficial for addressing emerging adults' CCA. In fact, since the majority of our sample reported significant worries in their open-ended responses but scored low on the measure of CCA, community-based approaches focusing on building agency may be most appropriate, with avenues to connect those with greater mental health needs to clinical services as needed.

\section{Limitations}

Although this study provides important contributions to the literature, there are a number of limitations that should be noted. First, we drew on cross-sectional data and therefore can only describe associations between constructs and not directionality. For example, it is possible that those who are experiencing mental health distress are more vulnerable to the impairing aspects of CCA and have reduced capacity to engage in collective action. Additionally, there were several limitations to the measure used to assess individual climate action, including that some items involved emotional as well as action-related responses and that the measure did not include the full range of individual actions that participants described in their open-ended responses. Future research is needed to develop more comprehensive measures of individual action to address climate change and to investigate whether a more comprehensive measures may yield difference results. This study also utilized a small, self-selected, non-representative student sample, with those more invested in addressing climate change likely self-selecting into the study. Data were collected in the context of the COVID-19 pandemic and remote learning at most universities, which may have contributed to heightened levels of distress. Future research should examine these constructs in more diverse populations, particularly with respect to race, geography, and education-level, as well as within clinical or treatmentseeking samples. Finally, while the qualitative data included provided a deeper understanding of participants' experiences than the quantitative data alone, in-depth interviews or focus groups would allow for greater insight into their experiences of CCA. Despite these drawbacks, the current study provides an important step towards understanding CCA among emerging adults and how it relates to clinical measures of MDD and GAD, as well as pointing to the potential of collective action to contribute to the well-being of those experiencing $\mathrm{CCA}$.

Funding The third author benefitted from a FIAS fellowship at the Paris Institute for Advanced Study (France). It has received funding from the European Union's Horizon 2020 research and innovation programme under the Marie Sklodowska-Curie grant agreement No 945408, and from the French State programme "Investissements d'avenir", managed by the Agence Nationale de la Recherche (ANR11-LABX-0027-01 Labex RFIEA+).

\section{Declarations}

Conflict of Interest On behalf of all authors, the corresponding author states that there is no conflict of interest.

\section{References}

Albright, J. N., \& Hurd, N. M. (2021). Activism, social support, and trump-related distress: Exploring associations with mental health. Journal of Diversity in Higher Education. Advance online publication. https://doi.org/10.1037/dhe0000316.

Alisat, S., \& Riemer, M. (2015). The environmental action scale: Development and psychometric evaluation. Journal of Environmental Psychology, 43, 13-23. https://doi.org/10.1016/j.jenvp. 2015.05.006

American Psychiatric Association. (1994). Diagnostic and statistical manual of mental disorders (4th ed.). Author.

Benoit, L., Thomas, I., \& Martin, A. (2022). Review: Ecological awareness, anxiety, and actions among youth and their parents - a qualitative study of newspaper narratives. Child and Adolescent Mental Health, 27, 47-58. https://doi.org/10.1111/camh.12514. 
Boehnke, K., \& Wong, B. (2011). Adolescent political activism and long-term happiness: A 21-year longitudinal study on the development of Micro- and macrosocial worries. Personality and Social Psychology Bulletin, 37(3), 435-447. https://doi.org/10.1177/ 0146167210397553

Braun, V., \& Clarke, V. (2006). Using thematic analysis in psychology. Qualitative Research in Psychology, 3(2), 77-101. https://doi.org/ 10.1191/1478088706QP063OA

Braun, V., Clarke, V., Boulton, E., Davey, L., \& McEvoy, C. (2020). The online survey as a qualitative research tool. International Journal of Social Research Methodology, 1-14. https://doi.org/ 10.1080/13645579.2020.1805550.

Burke, M., González, F., Baylis, P., Heft-Neal, S., Baysan, C., Basu, S., \& Hsiang, S. (2018). Higher temperatures increase suicide rates in the United States and Mexico. Nature Climate Change, $8(8), 723-729$

Canady, V. A. (2019). APA annual stress survey finds 2020 election one major cause. Mental Health Weekly, 29(43), 6-7. https:// doi.org/10.1002/mhw.32126

Chen, C. W., \& Gorski, P. C. (2015). Burnout in social justice and human rights activists: Symptoms, causes and implications. Journal of Human Rights Practice, 7(3), 366-390. https://doi. org/10.1093/jhuman/huv011

Cianconi, P., Betrò, S., \& Janiri, L. (2020). The impact of climate change on mental health: A systematic descriptive review. Frontiers in Psychiatry, 11, 74. https://doi.org/10.3389/fpsyt.2020. 00074

Clayton, S. (2020). Climate anxiety: Psychological responses to climate change. Journal of Anxiety Disorders, 74, 102263. https:// doi.org/10.1016/j.janxdis.2020.102263

Clayton, S., \& Karazsia, B. (2020). Development and validation of a measure of climate change anxiety. Journal of Environmental Psychology, 69, 101434. https://doi.org/10.1016/j.jenvp.2020. 101434

Clayton, S., Manning, C. M., Speiser, M., \& Hill, A. N. (2021). Mental health and our changing climate: Impacts, inequities, responses. American Psychological Association, and ecoAmerica.

Corral-Verdugo, V., Tapia-Fonllem, C., \& Ortiz-Valdez, A. (2015). On the relationship between character strengths and sustainable behavior. Environment and Behavior, 47(8), 877-901. https://doi. org/10.1177/0013916514530718

DeAngelo, L., Schuster, M. T., \& Stebleton, M. J. (2016). California DREAMers: Activism, identity, and empowerment among undocumented college students. Journal of Diversity in Higher Education, 9(3), 216-230. https://doi.org/10.1037/dhe0000023

Doherty, T. (2016). Theoretical and empirical foundations for Ecotherapy. In Ecotherapy: Theory, Research and Practice (Eds. Jordan, M. \& Hinds, J.) Palgrave, pp. 12-31.

Doherty, T. J., \& Clayton, S. (2011). The psychological impacts of global climate change. The American Psychologist, 66(4), 265276. https://doi.org/10.1037/a0023141

Eisenman, D., McCaffrey, S., Donatello, I., \& Marshal, G. (2015). An ecosystems and vulnerable populations perspective on solastalgia and psychological distress after a wildfire. EcoHealth, 12(4), 602-610. https://doi.org/10.1007/s10393-015-1052-1

Flanagan, C., Syvertsen, A., \& Stout, M. (2007). Civic engagement models: Tapping adolescents' civic engagement (Circle Working Paper 55). Medford, MA: The Center for Information and Research on Civic Learning and Engagement (CIRCLE)

Franklin, V. P. (2021). The young crusaders: The untold story of the children and teenagers who galvanized the civil rights movement. Beacon Press.

Goldmann, E., \& Galea, S. (2014). Mental health consequences of disasters. Annual Review of Public Health, 35(1), 169-183. https:// doi.org/10.1146/annurev-publhealth-032013-182435
Gong, F., Xu, J., Fujishiro, K., \& Takeuchi, D. T. (2011). A life course perspective on migration and mental health among Asian immigrants: The role of human agency. Social Science \& Medicine, 73(11), 1618-1626. https://doi.org/10.1016/j.socscimed.2011. 09.014

Gorski, P. C. (2019). Fighting racism, battling burnout: Causes of activist burnout in US racial justice activists. Ethnic and Racial Studies, 42(5), 667-687. https://doi.org/10.1080/01419870.2018. 1439981

Hayes, A. F. (2017). Introduction to mediation, moderation, and conditional process analysis: A regression-based approach. Guilford publications.

Hayes, K., Blashki, G., Wiseman, J., Burke, S., \& Reifels, L. (2018). Climate change and mental health: Risks, impacts and priority actions. International Journal of Mental Health Systems, 12, 28. https://doi.org/10.1186/s13033-018-0210-6

Henin, A., \& Berman, N. (2016). The promise and peril of emerging adulthood: Introduction to the special issue. Cognitive and Behavioral Practice, 23(3), 263-269. https://doi.org/10.1016/j. cbpra.2016.05.005

Heo, S., Lee, W., \& Bell, M. L. (2021). Suicide and associations with air pollution and ambient temperature: A systematic review and meta-analysis. International Journal of Environmental Research and Public Health, 18(14), 7699.

Hope, E. C., Velez, G., Offidani-Bertrand, C., Keels, M., \& Durkee, M. I. (2018). Political activism and mental health among black and Latinx college students. Cultural Diversity and Ethnic Minority Psychology, 24(1), 26-39. https://doi.org/10.1037/ cdp0000144

Hrabok, M., Delorme, A., \& Agyapong, V. (2020). Threats to mental health and well-being associated with climate change. Journal of Anxiety Disorders, 76, 102295. https://doi.org/10.1016/j.janxdis. 2020.102295

IBM Corp. (2020). IBM SPSS statistics for windows, version 27.0. IBM Corp.

Kessler, R. C., Berglund, P., Demler, O., Jin, R., Merikangas, K. R., $\&$ Walters, E. E. (2005). Lifetime prevalence and age-of-onset distributions of DSM-IV disorders in the National Comorbidity Survey Replication. Archives of General Psychiatry, 62(6), 593-602. https://doi.org/10.1001/archpsyc.62.6.593

Kleres, J., \& Wettergren, A. (2017). Fear, hope, anger, and guilt in climate activism. Social Movement Studies, 16(5), 507-519. https:// doi.org/10.1080/14742837.2017.1344546

Kovan, J. T., \& Dirkx, J. M. (2003). "Being called awake": The role of transformative learning in the lives of environmental activists. Adult Education Quarterly, 53(2), 99-118. https://doi.org/ $10.1177 / 0741713602238906$

Kroenke, K., \& Spitzer, R. L. (2002). The PHQ-9: A new depression diagnostic and severity measure. Psychiatric Annals, 32, 509-521. https://doi.org/10.3928/0048-5713-20020901-06

Kroenke, K., Spitzer, R. L., Williams, J. B., \& Löwe, B. (2009a). An ultra-brief screening scale for anxiety and depression: The PHQ4. Psychosomatics, 50(6), 613-621. https://doi.org/10.1176/appi. psy.50.6.613

Kroenke, K., Strine, T. W., Spitzer, R. L., Williams, J. B., Berry, J. T., \& Mokdad, A. H. (2009b). The PHQ-8 as a measure of current depression in the general population. Journal of Affective Disorders, 114(1-3), 163-173.

Lorimer, K., Knight, R., \& Shoveller, J. (2020). Improving the health and social wellbeing of young people: exploring the potential of and for collective agency. Critical Public Health. https://doi.org/ 10.1080/09581596.2020.1786501

McCreary, D. R., \& Sasse, D. K. (2000). An exploration of the drive for muscularity in adolescent boys and girls. Journal of American College Health, 48, 297-304. 
McGinn, M. (2019). 2019's biggest pop-culture trend was climate anxiety. Grist magazine. https://grist.org/politics/2019s-bigge st-pop-culture-trend-was-climate-anxiety/

Nairn, K. (2019). Learning from young people engaged in climate activism: The potential of collectivizing despair and Hope. YOUNG, 27(5), 435-450. https://doi.org/10.1177/1103308818 817603

Ojala, M. (2012). Hope and climate change: The importance of hope for environmental engagement among young people. Environmental Education Research, 18(5), 625-642. https://doi.org/10.1080/ 13504622.2011.637157

Palinkas, L. A., O’Donnell, M. L., Lau, W., \& Wong, M. (2020). Strategies for delivering mental health services in response to global climate change: A narrative review. International Journal of Environmental Research and Public Health, 17(22), 8562.

Randall, A. (2015). Mobilizing action on climate change and migration: The UK migration and climate change coalition. In Organizational perspectives on environmental migration (pp. 177-184). Routledge.

Reisner, S. L., Katz-Wise, S. L., Gordon, A. R., Corliss, H. L., \& Austin, S. B. (2016). Social epidemiology of depression and anxiety by gender identity. The Journal of Adolescent Health : Official Publication of the Society for Adolescent Medicine, 59(2), 203208. https://doi.org/10.1016/j.jadohealth.2016.04.006

Reyes, M. E. S., Carmen, B. P. B., Luminarias, M. E. P., Mangulabnan, S. A. N. B., \& Ogunbode, C. A. (2021). An investigation into the relationship between climate change anxiety and mental health among gen z filipinos. Current Psychology: A Journal for Diverse Perspectives on Diverse Psychological Issues. https://doi.org/10. 1007/s12144-021-02099-3

Riemer, M., Lynes, J., \& Hickman, G. (2014). A model for developing and assessing youth-based environmental engagement programmes. Environmental Education Research, 20(4), 552-574. https://doi.org/10.1080/13504622.2013.812721

Searle, K., \& Gow, K. (2010). Do concerns about climate change lead to distress? International Journal of Climate Change Strategies and Management. https://doi.org/10.1108/17568691011089891

Spitzer, R. L., Kroenke, K., Williams, J. B., \& Löwe, B. (2006). A brief measure for assessing generalized anxiety disorder: The GAD-7. Archives of Internal Medicine, 166(10), 1092-1097. https://doi. org/10.1001/archinte.166.10.1092
Stanley, S. K., Hogg, T. L., Leviston, Z., \& Walker, I. (2021). From anger to action: Differential impacts of eco-anxiety, eco-depression, and eco-anger on climate action and wellbeing. The Journal of Climate Change and Health, 1, 100003. https://doi.org/10. 1016/j.joclim.2021.100003

Taylor, S. (2020). Anxiety disorders, climate change, and the challenges ahead: Introduction to the special issue. Journal of Anxiety Disorders, 76, 102313. https://doi.org/10.1016/j.janxdis.2020. 102313

Tyson, A., Kennedy, B., \& Funk, C. (2021). Gen Z, millennials stand out for climate change activism, Social Media Engagement With Issue. Pew Research Center.https://www.pewresearch.org/scien ce/2021/05/26/local-impact-of-climate-change-environmentalproblems/

Velez, B. L., \& Moradi, B. (2016). A moderated mediation test of minority stress: The role of collective action. The Counseling Psychologist, 44(8), 1132-1157. https://doi.org/10.1177/00110 00016665467

Veronese, G., Pepe, A., Jaradah, A., Murannak, F., \& Hamdouna, H. (2017). "We must cooperate with one another against the enemy": Agency and activism in school-aged children as protective factors against ongoing war trauma and political violence in the Gaza strip. Child Abuse \& Neglect, 70, 364-376. https://doi.org/10. 1016/j.chiabu.2017.06.027

Wang, Z. H., Yang, H. L., Yang, Y. Q., Liu, D., Li, Z. H., Zhang, X. R., et al. (2020). Prevalence of anxiety and depression symptom, and the demands for psychological knowledge and interventions in college students during COVID-19 epidemic: A large crosssectional study. Journal of Affective Disorders, 275, 188-193. https://doi.org/10.1016/j.jad.2020.06.034

Wullenkord, M., Tröger, J., Hamann, K. R., Loy, L., \& Reese, G. (2021). Anxiety and climate change: A validation of the climate anxiety scale in a German-speaking quota sample and an investigation of psychological correlates. PsyArXiv. https://doi.org/10. 31234/osf.io/76ez2.

Publisher's note Springer Nature remains neutral with regard to jurisdictional claims in published maps and institutional affiliations. 